\title{
Spiritual practice in mental health: a focus on yoga
}

\begin{abstract}
Depression in the global population continues to have a negative impact on the health of individuals. Data shows there is an increase in the use of anti-depressant pharmaceuticals for treating depression in particular for underserved and at-risk populations. This paper supports the practice of yoga as a form of treatment for depression. The authors evaluates current systemic research and randomized clinical trial studies that support the efficacy of healing benefits of yoga and their positive impact on decreasing depression. The authors also address the potential mechanism that can explain the observed benefits of yoga in relationship to depression.
\end{abstract}

Keywords: yoga, depression, holistic, anxiety, physical postures, sudarshan kriya yoga, imipramine, electroconvulsive therapy
Volume I4 Issue 2 - 202I

\author{
Steven J Szydlowski, Peter P Amato \\ Department of health, University of Scranton, USA
}

Correspondence: Dr. Steven J. Szydlowski, Professor and MHA Program Director, University of Scranton, 413 McGurrin Hall, Scranton, PA 185I0, USA, Tel 570 94I 4367; 570947 7766, Email steven.szydlowsk@scranton.edu

Received: February 19, 2021 | Published: March 18, 2021
Abbreviations: SKY, sudarshan kriya yoga; EVT, electroconvulsive therapy; IMN, imipramine; BDI, back depression inventory; HRSD, hamilton rating scale for depression

\section{Spiritual practice in mental health: a focus on yoga}

Spirituality contributes to one's overall health and well-being. Ensuring an individual's physical, psycho-social, emotional, and spiritual health is nurtured is essential for self-care and provider treatment plans. Having awareness of holistic aspects of one's health and well-being is essential for achieving and sustaining optimal health throughout one's life span. Spiritual practice needs to be part of a wellness and care plan. Salman \& Yi-Hui ${ }^{1}$ found that depression in older adults were more likely to perceive port health and lower spiritual well-being. Yoga as a spiritual practice can contribute to better health throughout one's lifespan.

The ancient practice of yoga is often associated with relaxation and the practice of silence, self-reflection, and the experience of one, the universal being, and numerous other descriptions. The practice of yoga originated in Eastern culture, and has recently become prevalent in Western society. Seekers can find yoga classes, studios, schools, and workshops offered throughout the world. However, yoga is not often associated with medical care, health care, or clinical treatment plans. There are individuals who practice yoga, work in health care, and have a close understanding to the relationship of yoga to health. The author reviews three articles that support the health benefits in addressing depression with yoga. The authors believe that yoga, as part of a customized, functional medical plan for individuals, is an integral part of one's journey back to health, maintenance of health, wellness, and ultimately holistic well-being.

The authors believe depression begins with the way a person feels about the relationship between their life and surroundings. Depression is verbally expressed as a negative version of the lived experience. The mental attitude, perception, and verbal dialogue of one who is said to be experiencing depression is generally one of unhappiness, sadness and a discontented life experience. This extreme experience is said to be about the inability to perceive a positive outcome of a situation through the mind and imagination. The perceived reality of one suffering from depression takes on many expressions and symptoms. The authors understand that the symptomology of depression has been recognized as a type of mental agitation at the level of the mind. Those who suffer, or are diagnosed with depression, often exhibit forms of physical restlessness or an inability to remain calm with a relaxed frame of reference. The authors have experienced people suffering from depression who reveal deep negativity, various levels of mental agitation, and irritability at different levels and times. Confusion, anxiety, and physical pain are often understood and recognized as further symptoms that have varying levels. The authors understand that this mental attitude, often unexplainable, also is exemplified through having a difficult time with concentration, revealing a very active mind, along with an inability to focus. This inability to be present with clarity, breeds negativity and fear, and often times, feelings of numbness, hopelessness or helplessness. This life experience often creates deeper feelings of worthlessness, a withdrawal from true living, and a general lack of interest. The authors understand that as symptoms continue and if depression deepens, dramatic shifts may occur in the personality and are displayed in appetite levels, energy patterns, low self- esteem and relationships with others. Mental obsession is often thought of as a pathological process with certain beliefs, fears and inhibitions as its underpinnings. Mood swings, thoughts of death or contemplations of suicide are expressed as aspects in defining this rampant disorder. Others express depression through immense sadness, an immobilizing and at times a "freeze", or confusion. A vengeful anger has also been suggested as a symptom revealed through the underlying fear and frustration, which develops over the inability to reconcile and be happy.

Depression is often defined as a mood disorder. The authors are fascinated that the Western world, with all its science and medicine, remains unsuccessful in determining the exact cause of depression. Depression is an emotional problem, which is rooted in spiritual, disconnect. A discussion of symptoms and/or root cause is necessary to reveal the foundations of Eastern philosophy's contributions towards easing depression. This restoration of wholeness and integration can be experienced through yoga.

Yoga, over the ages has been shown to reduce stress, and improve mood by providing a relaxation method that enables a person to view life through a different lens. When yoga is understood as union, or 
integration of mind-body and spirit, it allows thoughts, feelings, and emotions to work toward unifying the mind, intellect, and ego with life. Yoga can be wonderful medicine in revealing the stillness and unity of the person experiencing depression. Uebelacker \& Broughton $^{2}$ concluded that evidence suggests yoga can be used to treat depression, anxiety, and other related mental health issues.

The authors'yoga teaching experience provides a firsthand knowledge of the power of yoga as a potential transformation through depression. The liberation of yoga utilizes the physical postures (asana); keeping the body busy, while allowing a person to move away from the mind in an egoic attempt to perfect the pose. When the physical body is practicing yoga, breath work is also introduced as an opportunity to lengthen the body, and to enhance the length of space between thoughts in focusing on the pose. This attempt at moving away from thoughts (and ego) and moving toward union of body and mind, using breath as the vehicle for transformation, often allows the depression to diminish. A person often experiences the essential Self as non-depressed. One experiences original nature, unbiased by illusion, free from past or future negativity and fear. When a person can move beyond the mind, it serves as a vehicle to distance oneself from past demons of depression. Yoga can provide the individual an opportunity to experience the true self beyond negativity.

The authors had personal experiences with clients and others who experience yoga and claim to encounter the "higher Self". This enables one to go beyond depression and reveal the latent history or illusion they have been living. The origins of the 10,000-yearold yogic tradition are revealed to us as an unfolding or an opening to the true self. The author believes the root cause of depression, is non-yoga, or the dis-integration of the self, leading to a lack of meaning with regard to one's highest life purpose. Although oneness or an integrated state is man's essential state, often life does not allow one to be fully expressed in this manner. Yoga can be viewed as an incredible modality that awakens one's true potential through its actual "experience". Additionally, proper nutrition and diet further enhances the mind-body connection. It restores the memory of wholeness and cellular resonance, and can move one beyond the illusion of a fragmented and compartmentalized being, allowing a person to live fully and experience a zest for life.

\section{Depression in underserved populations}

Depression and mental health needs continue to play a significant role in the health of underserved and at-risk populations and is more prevalent in low-income individuals. ${ }^{3}$ Various attempts continue to address depression in vulnerable populations. In a recent study, community/academic partnerships were used to create awareness and treatment for underserved African American and White elders. ${ }^{4}$ The researchers found that community engagement for outreach to underserved populations is critical in developing effective communication and treatment models. Choi, et al., ${ }^{5}$ suggest that many underserved individuals who are homebound with limited access to financial resources and other life demands can benefit from coping mechanisms to address depression. A more comprehensive approach to treating mental health and depression in underserved populations may be found effective. Mind-body therapies and psychotherapeutic interventions for low-income women, second generation Latinas, and other vulnerable populations have been found effective. ${ }^{6,7}$ Depression low-income women can also lead to other adverse behaviors impacting heath. Fowles, et al. ${ }^{8}$ found that life stressors in this population may result in low diet quality, poor nutrition, and the need for dietary intervention for those during pregnancy. The evidence supports the need to identify and utilize new techniques for improving overall health and coping mechanisms.

\section{Yoga and its relationship to depression}

The classic Patanjali eight limbs of yoga, when practiced, can reveal the actual "lived experience" of yoga or integration and awareness of wholeness. When the lived experience of being in the present moment beyond one's thoughts occurs, (this is yoga), the illusion or forms of thought, which distort a present moment reality, are overshadowed by the direct experience of the presence of one's higher Self. This allows one to continue to distance the Self from reality and permits one to recognize who they are at the core of their being. Depression results in not experiencing one's self from this position. This internal, yet interpersonal intervention can result in a state of mindfulness or the experience of full awareness. The authors have experienced participants embody a silent joy without cause when experiencing the gift and joy of yoga. Very often, it is the limiting thoughts or the narrow-mindedness of the individual, which blocks the revelation of a deeper and more positive experience. Yoga practice allows for a broader life experience in realizing that the demons of the mind or self-defining beliefs which inhibit a complete sense of self, are self-defeating and limiting. Yoga or union allows one to experience a broader opening to Life.

In writing this research paper, it is apparent that limited randomized control trials exist. There is much needed research in the area of yoga and depression. In the following section, the author reviews three randomized control trials. The analysis provided below will also show the limitations of each published article. Pilkington et al., ${ }^{9}$ provides an excellent systemic review of the research evidence on yoga and depression.

It is also important to note that yoga is often defined broadly, so the authors will describe the yoga intervention for each of the three articles. In the broad sense, it is the authors' experience that most people in Western culture perceive yoga as a physical exercise, stretching, or a workout as part of a fitness regime. Common types of this yoga are called power yoga or hot yoga, which demands intense heat and sweating. Yoga has also been ingrained to some extent as a social fad, trendy, and something to do as an activity. Having spent numerous years learning, practicing, and teaching what is considered more meditative yoga, or knowing the union of yoga, the can clearly discern the intention of the yoga type. While there are physical benefits to the "exercise" types of yoga, the author feels that the more meditative types of yoga, which focuses on the breath and its role in health and healing are essential and reflects yoga in its truest form. The authors attempted to identify the three articles that defined yoga more closely to breath as opposed to stretching and exercise. In particular, the authors identified three randomized clinical trial studies that used yoga to address depression.

Broota \& Dhir ${ }^{10}$ focused the research efforts using Broota's Relaxation, which included deep breathing, bow asana, elevation of legs, and cycling, supported with autosuggestion. This first treatment took place over three consecutive days for 20 minutes each. The second treatment was a brief 20 minute progressive relaxation technique. The control group did not receive the treatment. The authors selected this article because it was one of the first attempts to assess the impact of yoga on depression using a randomized clinical trial. 
The researchers obtained a sample of 30 outpatient clients diagnosed with clinical depression. Broota \& Dhir ${ }^{10}$ further define the sample in the study as a clinical diagnosis of depression primarily characterized as neurotic-depressive or reactive-depressive. The sample of 30 outpatient clients were then divided into 10 per group with age ranges from 19-48years. These inclusion criteria also contained individuals who were on medication for less than a year with no physical or mental damage.

Broota \& Dhir ${ }^{10}$ used 26 common symptoms of depression from three sources: DSM-III, ICD-9, and a pilot study. The assessment measures were done pre-test and post-test for both the treatment group and the control group. The findings show statistical significance in the reduction of depressive symptoms because of the yoga intervention. Another observation made in the article was that individuals who did not routinely exercise displayed tiredness and were often out of breath.

The authors suggests that the findings would have been enhanced if the researchers compared pre-test analysis between the treatment group and the control group. It is also the author's opinion that the study could have been lengthened to add more credibility to the results. As a yoga master, it is the authors' experience that, sustained yoga practice and intervention is required to maintain a level of calm and peace. Only then will individuals with depression truly feel the positive, long-term effects of yoga in alleviating depression. An individual with only three yoga sessions will not experience the integral benefits of yoga. In a final comment of this randomized control trial, the authors would suggest that future studies examine in detail the history of the use of yoga by the participants in the sample to have better determine one's connection to yoga.

Janakiramaiah et al., ${ }^{11}$ supports the importance of breath and breathing regulation at different rates in addressing depression. The sample size for this study was 45 hospitalized patients, which provided a little more strength in the results than the research study reviewed above based on the larger sample. The researchers divided the sample of 45 participants into groups of 15. The treatment group received Sudarshan Kriya Yoga (SKY), which included approximately 30 minutes of rhythmic hyperventilation followed by 15 minutes of normal breathing. This process was done once daily, for 6 days for four consecutive weeks. The intent of the research was to understand the efficacy of SKY compared to electroconvulsive therapy (EVT) and imipramine (IMN). The control group received only the EVT and IMN. Janakiramaiah et al.," ${ }^{11}$ identified the inclusion criteria as hospitalized individuals with a DSM-IV diagnosis of melancholic depression who were not treated for the current episode, but were deemed as medically fit.

Similar to the first article review, pre-test and post-test measures assessment timeframes were used in addition to weekly assessments. The instruments used in the study were the Back Depression Inventory (BDI) scale and the Hamilton Rating Scale for Depression (HRSD). Ultimately, the finding showed that reduction in the scores for each scale did occur successively on occasions in the treatment group, but did not show significant differences in the control group. The researchers' conclusion for the study is that SKY can be a mechanism of treatment for individuals diagnosed with melancholic depression, but does not show a greater efficacy than ECT and IMN. The author suggests that further studies in this area could review the use of SKY, ECT, and IMN as an integrative yoga therapy to address the population of study.
The authors feel that this research article did a better job identifying the sample population characteristics than the first article, but still did not address the prior history of the samples use of yoga. As with the first review, the authors strongly believe that this would play a major role in any yoga intervention study. This would be a good topic for further research.

The final randomized clinical trial study the authors reviewed targeted a different sample population. The researchers obtained a sample of 50 female university students that were diagnosed with severe depression. Of the 50 part pants, 25 received the yoga intervention and 25 did not receive any intervention as the control group. Khumar et al., ${ }^{12}$ identified the diagnosis by using the Amritsar Depression Inventory, the Zung Depression Self Rating Inventory, and interviews with the women who ranged from the ages of 20-25years. The students did not present other medical conditions and were not receiving any other treatment. The researchers also include in the study criteria that participants needed to have depression for 2-3months.

Khumar et al., ${ }^{12}$ identifies Shavasana yoga as the intervention used in the study that focused on rhythmic breathing and relaxation. The intervention was used in the treatment group for 30 minutes daily for 30 days. The authors will discuss the findings of the controlled study, but feels it is important to note that the sample size of 25 in both the treatment group and control group, limits the level of strength in the study. In most, if not all of the randomized clinical trials using yoga for depression, have small sample sizes. This is even true from the author's review of the literature on yoga and other illnesses. A largescale randomized clinical trial in this area is needed.

Khumar et al., ${ }^{12}$ found significant differences in pre-post scores for the yoga group and significant differences between the treatment group and control group at mid and post-treatment. The authors believe that more detail of the participants would provide more insight to the reader. There was limited information on the participants beyond the baseline data collected on the pre-test. In summary, the article reveals positive impacts of yoga on alleviating depression. The researchers also suggest that the longer the use of the yoga intervention, the more positive impact it can have on treating women with depression.

\section{Yoga as treatment}

The basis for the authors' explanation of how the mechanism of action, yoga, breath, and body alignment, is grounded in the peerreviewed articles summarized above, and the authors' personal training and experience with yoga. Klainin-Yobas, et a., ${ }^{13}$ identified mindfulness-based interventions are efficacious in treating depressive symptoms in adults with mental disorders and could be integrated into care plans. Current findings continue to support the use of yoga and mindfulness for treating mental health and addictive behaviors..$^{14,15}$ The reader should recognize, traditional research methods used to connect yoga to the one's health benefits in controlled settings is often challenging because it does not address the individual uniqueness of the person's experience. The authors suggest this from personal experience, along with the emerging concept of functional medicine or individualized health as discussed in the mind body program. The authors believe yoga, or the union of yoga, is very difficult to measure from a traditional sense. However, the benefits are real.

The authors view the journey of depression through the experience of yoga, similar to the metaphors of "peeling an onion" or "melting of 
an ice cube". The history or lineage of yoga as revealed and experienced by many tells us that the human endeavor of moving from darkness to light involves moving through layers of personhood known as koshas, or thought of, as sheaths. It is the authors' opinion that many individuals who experience chronic or acute depression attempt to find treatment externally in the form of counseling, prescription, or some other mechanism. The authors are not here debating the efficacy or benefits of those mechanisms, but simply suggesting that through yoga, individuals can heal from within.

The first mechanism of action is to recognize that one views physical life and living metaphorically as a primary food, or only experiences life at a dull level of experience or knowingness. The authors recognize that yoga postures and breathing techniques cognized from antiquity in the school of Hatha yoga, can move one to an experience beyond thought. The advanced mechanisms attained if one practices yoga regularly could provide an even deeper relationship with prana or energy in experiencing a deeper sheath, or layer of existence. The practice in moving beyond thought in experiencing the quiet mind is the largest step in experiencing the self beyond the attachment to the story. Moving further in absorption, practice and refinement, the next mechanism, which arrives for the individual, is spoken of as a "knowing", or an experience of the present moment, a quiet mind beyond thought. This experience of existence can be removed from the relationship with depression and symptomology. It is the author's opinion that eliminating the thoughts and patterns that created the depression through the practice of yoga or stillness is an effective mechanism.

The advanced yogic mechanisms of breathing techniques and meditation have been reported as true rapture, bliss, or ecstasy. The history of yoga recognizes this "peeling away" of illusion in experiencing what is referred to as the True Self over time through the unveiling of the sheaths of illusion known as the "five koshas". The authors who have taken the journey, feels strongly that the true reality which unfolds through the koshas is the most powerful and natural medicine known to man. Man is this medicine and needs to awaken from suppression and depression through yoga postures, conscious breath work and meditation. Often time individuals blame external sources such as the environment, the relationship with another, one's job, or chaos in general that one perceives life bringing that causes one's depression. This "peeling away" of illusion that is fabricated by one's thoughts can address the root cause of the depression and allow individuals to turn inward by focusing on breath.

Yoga and conscious breathing is a modality to address depression and can be a very powerful healing, and calming technique. Since the practice requires no batteries, medications, or further instruction, health practitioners can utilize this simple method for stress reduction and a technique to curb one's depression. Conscious connected breathing allows one to cultivate a sense of mindfulness. Becoming aware of awareness is central to healing.

\section{Conclusion}

The authors recognize that the exact cause of depression may never be known, and concludes that when man takes the journey of yoga, the experience of this ultimate reality embodies the consciousness of the intention, which cannot be depressed in experiencing the rapture and oneness of who we truly are. This is truly a beyond words experience, which quantum science recently has begun to discuss. The experience cannot be measured, and therefore is restricted only by the attention given to the energy of change which imparts yoga can alleviate depression, experience the journey. It is how one experiences the journey.

There has been an increase in the number of studies that investigate the health effects of yoga. However, research on yoga is in an introductory stage, even with the increasing global popularity of yoga. Based on the review conducted in this paper, the authors' personal experiences, and the observed benefits of yoga, further research and more quality studies with larger sample sizes will help to bring yoga to the forefront of practitioner's treatment plans, specifically in helping individuals with depression back to optimal health.

Further research is required in the area of longitudinal benefits of yoga to address depression. The authors found limited articles that measured long-term health impact of a daily yoga practice and its effect on sustaining decreased depression in individuals. However, given the popularity in Western culture of yoga, yoga classes, workshops, and studios, the author feels the time is ripe to integrate research on the health benefits of yoga into mainstream clinical and wellness settings.

The authors strongly believe that for future research studies' attempting to measure the impact yoga has on alleviating depression, researchers should gather information on prior yoga practice experience by the participants. In fact, it would be an interesting study to address the question: do individuals that have a daily yoga practice have less depression and those who do not have a daily yoga practice? Of course, the research design, framework, sampling process, and methods would need to be created, but the findings would be interesting.

Another area that the authors feel further research is needed, is to measure the effects of various types of yoga on depression. In the literature review for this paper, which included studies that were approached from a randomized clinical trial, systemic review, pilot study, or some other methodology, it seems that variations of the term yoga were used in the body of literature. The authors feel that to compare the effects of yoga on depression, or any other illness or disease, longitudinal studies using a specific type of yoga are needed. For example, the use of SKY in alleviating depression, or the use of Hatha yoga in alleviating depression, or the use of Power yoga in alleviating depression should be studied. However, the authors do appreciate any research that is being conducted on the benefits of yoga, but more focused and defined yoga interventions in a study should be used to advance to the research so comparative analysis and findings can occur.

Overall, the authors feels that health care providers and the medical community as a whole need to embrace yoga as a treatment for acute and chronic conditions, in this case, depression. Once this occurs, the total population from which samples could be drawn would be larger and lend itself to more longitudinal research studies. This would also provide increased clinical settings for the research to occur.

The practice of yoga offers the potential to move beyond the mind, intellect, and ego and give a direct experience of an expanded state of awareness, or consciousness. Union, or integration toward our essential state is a fulfilling experience and recognition that all is well and healing can occur through a profound knowingness. This expanded awareness has deep societal as well as personal benefit as man recognizes today that only through a change in human consciousness, can the world be transformed. 
Based on this understanding and knowingness of yoga, the authors suggest that researchers have and will continue to have an intense challenge in trying to measure the subjective nature and intangible aspect of yoga. To further develop this thought, there is an increased challenge in measuring an individual's consciousness or an expanded state of awareness, let alone trying to relate this in a cause and effect relationship to depression. It is the authors' opinion that the traditional approach to research and measurement cannot fully individualize, from a functional medicine standpoint, the health benefits one obtains from yoga as it relates to depression. It is unique to each person. However, traditional research approaches do and can support the benefits of yoga in a broad sense and the need to integrate yoga interventions into a patient's treatment and wellness plan.

\section{Acknowledgments}

None.

\section{Conflicts of interest}

There are no any conflicts of interest.

\section{Funding}

None.

\section{References}

1. Salman A, Yi-Hui L. Spiritual practices and effects of spiritual wellbeing and depression on elder's self-perceived death. Appl Nurs Res. 2019;48:68-74.

2. Uebelacker LA. Broughton MK. Yoga for depression and anxiety: A review of published research and implications for healthcare providers. $R$ I Med J. 2016;99(3):20-22.

3. Bowser DM, Utz S, Glick D, et al. A systematic review of the relationship of diabetes mellitus, depression, and missed appointments in a lowincome uninsured population. Arch Psychiatr Nurs. 2010;24(5):317-329.

4. Dobransky-Fasiska D, Brown C, Pincus HA, et al. Developing a community-academic partnership to improve recognition and treatment of depression in underserved African American and White elders. Am J Geriatr Psychiatry. 2009;17(11):953-964.
5. Choi NG, Hegel MT, Sirrianni L, et al. Passive coping response to depressive symptoms among low-income homebound older adults: Does it affect depression severity and treatment outcomes? Behav Res Ther 2012;50(11):668-674.

6. Levy LB, O'Hara MW. Psychotherapeutic interventions for depressed, low-income women: A review of the literature. Clin Psychol Rev. 2010;30(8):934-950.

7. Heilemann MV, Pieters HC, Kehoe P, et al. Schema therapy, motivational interviewing, and collaborative-mapping as treatment for depression among low income, second generation Latinas. $J$ Behav Ther Exp Psychiatry. 2011;42(4):473-480.

8. Fowles ER, Stang J, Bryant M, et al. Stress, depression, social support, and eating habits reduce diet quality in the first trimester in low-income women: A pilot study. J Acad Nutr Diet. 2012;112(10):1619-1625.

9. Pilkington K, Kirkwood G, Rampes H, et al. Yoga for depression: The research evidence. $J$ Affect Disord. 2005;89(1-3):13-24.

10. Broota A, Dhir R. Efficacy of two relaxation techniques in depression. Journal of Personality and Clinical Studies. 1990;6(1:83-90.

11. Janakiramaiah $\mathrm{N}$, Gangadhar $\mathrm{BN}$, Naga venkatesha PJ, et al Anitdepressant efficacy of Sudarshan Kriya Yoga (SKY) in melancholia: A randomized comparison with electroconvulsive therapy (ECT) and imipramine. J Affect Disord. 2000;57(1-3):255-259.

12. Khumar SS, Kaur P, Kaur S. Effectiveness of Shavasana on depression among university students. Indian Journal of Clinical Psychology. 1993;20(2):82-87.

13. Klainin-Yobas Cho MAA, Creedy D. Efficacy of mindfulness-based interventions on depressive symptoms among people with mental disorders: A meta-analysis. International Journal of Nursing Studie. 2012. p. 109-121.

14. Khanna S, Greeson JM. A narrative review of yoga and mindfulness as complementary therapies for addiction. Complement Ther Med. 2013;21(3):244-252.

15. Witkiewitz K, Bowen S. Depression, craving, and substance abuse use follows a randomized trial of mindfulness-based relapse prevention. $J$ Consult Clin Psychol. 2010;78(3):362-374. 